\title{
A STUdy On Data STREAMing In Fog CoMPUTING ENVIRONMENT
}

\author{
Shymaa Mohammed Jameel ${ }^{1}$ \\ ${ }^{1}$ Institute of Informatics for Postgraduate Studies Iraqi \\ Commission for Computers and Informatics, Baghdad, Iraq \\ shymaa792003@yahoo.com
}

\author{
Dr. Muayad Sadik Croock ${ }^{2}$ \\ ${ }^{2}$ Computer Engineering Department, \\ University of Technology, Baghdad, Iraq \\ 120102@uotechnology.edu.iq
}

\begin{abstract}
In lately years, data streaming is become more important day by day, considering technologies employed to serve that manner and share number of terminals within the system either direct or indirect interacting with them.

Smart devices now play active role in the data streaming environment as well as fog and cloud compatibility. It is affecting the data collectivity and appears clearly with the new technologies provided and the increase for the number of the users of such systems. This is due to the number of the users and resources available system start to employ the computational power to the fog for moving the computational power to the network edge. It is adopted to connect system that streamed data as an object. Those inter-connected objects are expected to be producing more significant data streams, which are produced at unique rates, in some cases for being analyzed nearly in real time. In the presented paper a survey of data streaming systems technologies is introduced. It clarified the main notions behind big data stream concepts as well as fog computing. From the presented study, the industrial and research communities are capable of gaining information about requirements for creating Fog computing environment with a clearer view about managing resources in the Fog.

The main objective of this paper is to provide short brief and information about Data Streaming in Fog Computing Environment with explaining the major research field within this meaning.
\end{abstract}

Keywords: Data Streaming, Fog Computing, Cloud Computing, Big Data, Quality of Service.

\section{INTRODUCTION}

The term fog is a side by side term used with cloud computing. The aim of fog technology is reducing the amount of data which requires being sent to the cloud to be processed, analyzed and stored. This type of technology generally, enhances the real-timeliness and effectiveness, via the migration of computing resources, services, and applications to network edges. [1]

Fog computing is a decentralized computing notion that doesn't entirely depend on a sole central component like the cloud [2], [3]. It's capable of overcoming the issue of high latency of cloud computing with the use of idle resources of a variety of devices around the users. None-the-less, fog computing is dependent on cloud for doing complicated processing. In contrast to cloud computing, fog utilizes numerous devices that surround us having computational ability. Presently, even a smart-phone of low-specification has computational ability, in some cases with multiple cores [4]. As a result, numerous devices such as base stations, smart phones, switches, routers, and other devices of network management equip with the storage capacity and processing power might play the role of Fog devices. The resources to those devices are idle outside the period of peak hours [5].

Within big data and big data stream, approaches of mining might be implemented with important information that comes from a variety of sources of data, such as full-fledged devices and sensor-like gadgets (such as laptops and tablets). In addition to that, those new scenarios give the ability of offering part of a solution to the most widely spread issues of legacy that are related to environments of distance learning and educational approaches of data mining [6].

\section{STREAMS OF BIG DATA}

Big data streams are rapidly turning into one of the major paradigms in data science area [7]. They emerge in an everincreasing variety of areas-web, intelligent systems, sensors, and many others. Typically, it is produced with the use of modern platforms involving numerous applications, devices, and users. Certain technologies and approaches are necessary for dealing with non-stationary data, virtually infinite streams, and enhancing knowledge pieces [8].

Big data streams have a number of ideas and issues in common with the concept of big data [9], like velocity, variety, and volume. In fact, big data and big data streams are identified with massive amounts of information, that are created at fast rates, and that could be having unknown structures [10]. The basic dissimilarities those two paradigms are associated with the fact that big data streams are generated at faster rates, could be produced by a number of heterogeneous sources and require being analyzed nearly immediately. In fact, big data streams offer observations sequences that are temporally-ordered [11]. Which have the possibility of being time-stamped, with varying time scales, based on certain sources of data. None-the-less, observations ordering has a considerable amount of significance due to the fact that there could be an important inter-observation relation (in other words, time series), or due to the fact that underlying occurrence could change with time, in some cases abruptly (in other words, concept shift) or gradually (in other words, concept drift). Every one of the observations comprises a complicated data record. It's generated with a number of unspecified nature variables, probably acquired from heterogeneous streams and based on certain sources [12].

Throughout the past years, massive amounts of technologies and standards have been proposed to handle, manage and analyze big data [13]. Those methods have not been appropriate to handle streams of big data for the sake of extracting advantageous insights from big data streams. Actually, this type of methods is usually dependent on batch 
processing and focuses on actual data, instead of the provision of real-time dispatching and processing [14]. For the sake of dealing with streams of big data, approaches and technologies are required to design and implement costcompetitive and scalable services, which can actually capable sufficiently collect and preprocess streams of data. This is performed for the sake of extracting beneficial knowledge for exploiting in certain situation. Next, we will present a summary of the main critical matters which have to be dealt with in the cases of big data streams:

- The structure which is responsible of managing streams of big data have to be capable of working in real time and for providing responses that have low-latency. Usually, those features aren't considered by the technologies of big data, due to the fact that data are stored statically, while in the case of dealing with streams of big data, information progresses and ages over time [16].

- The rate of data generation is a significant demand which has to be considered, as well as the time which is important for the analysis of stream and for the exploitation of its data [15].

- Data flows have to be chosen with accuracy, they also have to preprocessed and analyzed for a variety of reasons and final users. Particularly, infrastructures of big data stream could require performing aggregation of data, pruning or filtering, with various granularity levels. This is performed for the sake of minimizing latency in the conveyance of ultimate output of computation to particular users and for the improvement of the precision of responses that are provided by them [17].

- Extraction of knowledge from streams of big data needs new methods for data mining to work in realtime and with inputs that are irregular and out-oforder. In fact, models have to be keep being updated for the sake of keeping up with novel advances, shifts, concept drifts, and re-occurrences [18].

\section{Fog Computing}

Big data streams, which come from linked devices, produce massive information amounts that may be further processed and utilized for building numerous convenient services to the users. Clouds, and their warehouse-like, rack-mounted, data centers, comprised big data natural collection environment, because it is scalable, robust and costeffective. None-the-less, Fig. (1) illustrates the way newest models are moving in the direction of different level hierarchy which plays a role in data collections. Amongst them, designers of system are capable of explicitly distinguishing fog layer, which is typically populated by smart gate-ways which are closer to the smart devices and sensors compared with cloud servers. Fog [19], [20] is a newly-emerged concept, which has the aim of extending services and elaboration of the cloud to network edge, via depending on proximity to the user. Dense physical distribution and mobility support which could be guaranteed for the devices, even when network connection is not available. Fog computing particularly indicates gateways, which are in fact are the ones referred to as fog nodes, whereas edge computing presumes that intelligence is placed further down, directly to end devices [21].

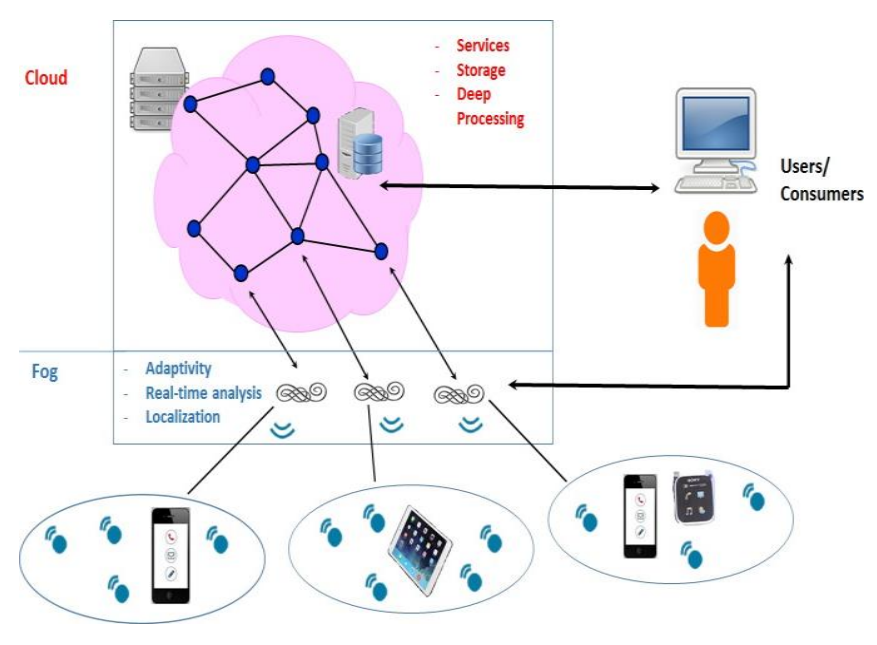

Fig1. Cloud and fog-based model [19]

In general, clouds remain a significant player in global architecture, mostly, this is attributed to the fact that it is capable of concentrating powerful data centers, in addition to providing a general outlook on the entire architecture. In addition to that, clouds might as well be changed to big data streams of the consumer, which come from layers of fog, in a way of hierarchy which could comprise several tiers of fog, in addition to inter/intra-layer communications. Each of fog and cloud might participate in communications with users, offering various, however, complementary services in continuous cooperation form [22]. Cloud might regulate large and historical data-sets and might be responsible for long and deep big data analyses, while fog might be focused on shorter times of responses, flow control, and localization information via empowering fog gateways with computing abilities. This might improve throughput, power savings and consolidating available resources as a result [23]. In addition to that, fog might be utilized in cases of failures or disasters of cloud, in a form of a distributed backup of that data which is stored in the actual cloud. Additionally, and more significantly, the use of fog diminishes data amount which is sent to cloud, thereby decreasing the amount of required band-width and raising data security, via avoiding the transfer of these pieces of information which could be risky when it comes to legal regulations and privacy [24].

None-the-less, fog nodes might pose a few disadvantages as well, in comparison with cloud, which need to be thoroughly considered:

- They cannot ensure continuous network connectivity.

- They can't be considered as reliable as cloud servers; in addition to that, the identification and repair of a fog node can be rather complicated.

- Heterogeneity could result in sharp resource differences amongst nodes and thereby hold back the cooperation. 
- Scheduling issues are complex, and there remains no $100 \%$ security for where to do operations like the balancing of workloads [25].

- Privacy and Security are a concern for smallresourced and low-powered nodes which could include sensitive data and might be easily assailable or which are capable of supporting various non-interoperable cryptographic standards.

- Mobility could be interrupting for executing processes on fog nodes, and other means for providing it seamlessly have to be later enacted.

- Consistency and redundancy of data could be difficult in the management, even in the case where the ultimate result might be more sufficient compared to the exclusive use of cloud.

- Management of data and discovery of cervices are difficult, like selecting the optimal algorithms for the sake of shuffling data amongst the devices. [26].

\section{DIFFERENCES BETWEEN CLOUD AND FOG}

Architecture of fog computing have been modeled on the basis of Fog clusters in which numerous Fog devices take part in cooperating with processing. However, data-centers are basic hardware cloud components. [27] as a result, cloud computing is computationally expensive, and it also consumes much energy. In comparison, operational costs and energy consumption in the concept of Fog computing are low [28]. Fog's location is closer to users, which is why the distance between Fog devices and end users might be a single or a few hops [29]. All agreed with distance between the cloud and end users, which is a multi-hop distance. Because of the distance, latency of communication for cloud is, at all times, high in comparison with Fog. [30] cloud is a more centralized method whereas Fog is more distributed, according to the physical orchestration [31].

There is no possibility for Real-time Interactions for clouds because of their high latency, however, this issue may be solved easily with the use of Fog. None-the-less, the failure rate in Fog is high, which is attributes to decentralized management, wireless connectivity, as well as power failure [32]. The majority of devices in the Fog are connected in wireless means due to the fact that handheld devices and smart gadgets take part in Fog systems [33]. The majority of those devices, as well as other devices of network management, are decentralized. Those devices might fail in the case where software isn't properly regulated. Users could unaware of malicious software which might result in device failures. In addition to that, fog processing might also fail in other cases, for instance, every Fog device permits its own application processing. Which is why, data application processing operations in Fog devices typically take $2^{\text {nd }}$ priority [34]. In the case where Fog device is entirely used by the actual device application, then it might fail in doing any Fog processing. As a result, scheduling resources and applications in Fog systems is usually more complicated [35]. Moreover, handling of failures in Fog systems is competitive due to power failures, which is only a problem due to the fact that devices are running on batteries [36].

\section{OVERVIEW OF STREAMING Fog COMPUTING SYSTEMS}

Platform based on cloud or fog changes rapidly, reasons for these changes are mainly reference to the huge revolution in the communication, cloud services, fog services and smart devices that affect the design of these platforms.

Many researchers develop systems in data streaming using fog computing and cloud computing which can be reviewed in this survey:

In [37] authors proposed architecture for the reduction of limitation in video out flowing with the use of fog technology for delivering real-time communication in addition to considerably shorter time of response, and the algorithm of weighted round robin (WRR) to schedule streaming tasks in fog. The experimental results have displayed the fact that in their proposed architecture the latency is minimal and decent video quality with variations in the band-width. The proposed system is client-server where the server is the computing node of the fog system. The initial step is a socket program which has utilized TCP/IP protocol for making communication amongst server and multiple clients. To extract and stream video frames, authors have presented streaming protocol which is similar to the works of the DASH technology, WRR has been utilized for the scheduling of streams of videos between the clients. The aim of this presented architecture was the execution of video streaming to each of the clients requests simultaneously and making the quality of videos vary based on the band-width of the client. The value of latency has been influenced by the varying of band-width of the client that decreases by high computations of fog systems and the increases in band-width.

In [38], authors highlighted some edge computing prospects for the interactive media, and have presented some initial researches in this area. They shed light on the way that edge computing may be utilized for tackling different issues that are encountered by modern applications of the interactive media. They have given advantages of the use of edge computing for saving energy, costs, and band-width in applications of multi-media and video stream. The basis for the concept of edge computing was stated on the basis of pushing storage and computation abilities to network edge. Those abilities may be accomplished via locating storage devices and servers at edge, like base stations, cloudlet (micro data center), and ISPs. They have as well improved the ability of the existing network devices for supporting storage and computational capabilities as well as the regular abilities, like it has been suggested in fog systems. Nonethe-less, the fundamental aims and concepts in all presented technologies, which are, mobile edge, fog, nano/micro datacenters, and cloudlets have been similar. Which is why, they have jointly referred to all of those technologies as "edge computing". The fundamental aim of edge systems was providing computational and storage facilities nearer to users' devices, and extend the services of the cloud as near as possible to users, for the facilitation of rapid responses and real-time services. A limited cloud services version might be located and operated at edge, in a way that requests of users didn't have the need of travelling to distant 
data centers of the cloud, and rather operated at the edge. This type of the model gave the ability of wide base of ubiquitous, context-aware, and real-time services.

The [39] introduced a quality of service-aware streaming via fog a computing infrastructure which has been suggested for relieving the delivery issues of conventional content via the adapting of videos to existing conditions of the network and probably utilizing local resources of computing. Fog computing has been modeled for extending cloud network edge for the sake of decreasing network congestion and latency. This system gave the ability for service providers in improving utilization of resources and QoS via including various layer information for the sake of delivering and adapting a video in the optimal possible quality over infrastructures of fog computing. There different Issues in the selection of Edge Nodes:

- Loading the approach of Edge Node Balancing: The aim of this approach is the evaluation of computing loading of each edge node in fog structures, at the same time as ensuring the provision of end device with a higher quality of streaming.

- The prediction of Available Band-width between the End Device and the Edge Nodes: The precision of predicting the band-width significantly influences the delivery of segments of content. The general approaches are normally based on recorded band-width for the prediction of future band-width.

Authors have proposed a quality of service-aware streaming service via the infrastructures of fog computing. They constructed communication and approaches of management amongst the components of the fog, including prediction of band-width and the loading of approaches of monitoring amongst nodes, and designs an approach of loading balance for nodes of the edge in infrastructures of fog. In experiments, the rate of errors and the delay time of end devices of a model of band-width prediction with a variety of bandwidth values have been discussed. Simultaneously, the operation elects of edge nodes balancing approach has been arisen.

In [40], authors took urban traffic monitoring to be a case study. An approach of dynamic video streaming has been suggested for meeting real-time decision making and information processing requirements. They studied the possibility of enabling the function of multi-target tracking with the use of a simpler single algorithm of tracking targets. A model has been generated and its efficiency has been assessed. It was a 3-tier architecture system of urban surveillance that has been made up of a layer of surveillance application (referred to as user layer as well), a layer of fog Computing, and a layer of cloud computing. The near-site or on-site, layer of fog computing has been the most important for real-time processing of data. Many different smart devices play the role of nodes of Fog Computing, which are smartphones, smart tablets, on-board computing devices on drones, or laptops in police cars. After collecting raw video streams, rather than transferring those streams to remote cloud centers, tasks of processing have been allocated to near-site devices of fog computing. As a result, latency of data transmission from the area of surveillance to Cloud center has been eliminated. Moreover, the layer of Fog Computing prevented local important data from being transmitted to Cloud. It decreases the communication network workload.

In [41], authors have proposed a platform of edge computing, in which nodes of mobile edge are hardware components that have been utilized on a transit bus in which descriptive analytics have been utilized for uncovering significant patterns from data streams of real-time transit. An experiment of application has been utilized for evaluating benefits and drawbacks of the presented structure for supporting descriptive analytics at a node of mobile edge and generating actionable information for transition of managers. The presented structure of edge computing has maintained scalability to the whole system of transit. In addition to that, it has prepared the path for the development of innovative analysis services at edge network in near future for the sake of solving issues of vastly-growing data which is generated by edge sensors and devices.

In [42], the authors have reported a computer simulation of operating mining algorithms of data streams in fog systems. In addition to that, selection of features, which is supported with swarm search, has been utilized as an approach of preprocessing for the enhancement of local Fog data analytics speed and accuracy. During the experiments, results have shown which algorithms have been the optimal options for the delivery of edge intelligence in the environment of fog computing. In the environment of Fog computing, data have been streamed in large amounts non-stop to the model data stream mining. Therefore, the framework has to be capable of handling incremental learning from seeing a part of data stream at a time only. In addition to that, it rapidly updated itself whenever it sees fresh data.

All the data streaming researches mentioned above try to provide a proper solution to the security concerns of the fog computing.

\section{Quality of Service Evaluating Criteria}

Different criteria can be considered in evaluating the required Quality of Services, including:

Scalability: A Fog system are predicted to cover a massive number of devices of IoT/end-user. In addition to that, they could include numerous fog domains, applications, and fog nodes. Some of the applications could as well have numerous components. A Fog system should be operational at those large scales. They need to scale up and down in an elastic way. The need for architectural modules in ensuring this scalability like the engine of elasticity.

Heterogeneity: A fog stratum and in cloud stratum nodes are quite heterogeneous from the point of view of the storage and computational abilities. Fog systems have to be capable of coping with this heterogeneity. Needing to consider heterogeneity in the case of determining the application component(s) that have to be utilized.

Management of the QoS: Fog systems are promising enablers for applications of real-time because of fog nodes proximity to devices of end-users/ IoT. None-the-less, latency considerably varies based on the location of components of the application. Quality of service has to be 
regulated. The requirement for architectural modules for Quality of service regulation like the engine of migration.

Mobility: devices of IoT/end-user and fog nodes may be mobile. Fog systems have to be capable of handling this mobility. The needs to have architectural modules like an engine of mobility for ensuring service continuity for endusers.

\section{COMPARING VIDEO STREAMING SYSTEMS}

Numerous criteria are used to compare video streaming systems in terms of Heterogeneity, Scalability, Mobility and QoS Management and other factors related to the system architecture and design. These criteria can be compared in summery as shown in Table (1).

\section{Problem Statement}

In this section a short review presented to the limitations of the current data streaming in fog computing systems that are preventing the deployment of the new data streaming scenario:

1) The IPv4 suffers from the well-known address depletion problem.

2) Challenge of processing the flood of data created every day.

3) Related security challenges, since fog is a distributed decentralized infrastructure.

4) Huge data mining and data analysis for today and yesterday data.

5) Amount of data entering for processing at the same time.

security issues related to the fog computing:

Many security concerns related to the use of fog computing such as:

- Provide secure communication to the connected nodes within the fog network to ensure the security.

- $\quad$ Authentication of people connected to the network and how they behave within this network.

- Authorization of the connected people and only authorized people access sensitive information.

- information confidentiality.

\section{SUGGESTED SOLUTIONS}

Since the limitation connected to data streams in fog computing, it is not associated with the fog computing concept or the data streaming itself. Some of these limitations can be avoided using the following solution suggestions:

1) Using IPv6 protocol that can solve the addressing issue with IPv4.

2) The processing of these data can be performed by applying the concept of big data on the data flooding.

3) Paying more attention to the security issues that are associated with fog computing and try to solve it.

4) Using the data science in analyzing the data on databases and applying the prediction algorithms to be up-to-date.
5) Scheduling for the data needs to be applied to ensure no conflicts or deadlock happen in the system and no starvation scenarios.

\section{CONClusion}

Using fog computing can decrease the amount of the data which need being uploaded into the cloud and move computational overhead to network edge. However, it contains a many drawback, such as requiring a specific maintenance level which is greater than the maintenance level of cloud solutions, the bigger number of operations of update are needed. In the case of simpler and more lightweight the uncertain savings level in costs of software and hardware. In the instances of student devices aren't compatible or usable, a particular level of data portability overhead when network collectors or local smart nodes fail and problems of security that are associated with devices that are battery-powered. Advantages of using in is sufficient utilization of resources merely interested fog gateways and local devices could be utilized. Full-fledged applications of mining of big data stream and energy efficiency, as light-weight low-power devices have been utilized. Many researchers try to provide fast, accurate and secure methods for data streaming they comparison between these researches is done via Media, Heterogeneity, Scalability, Mobility, QoS Management, Type of user Computation Element and Scheduling these factors will affect the quality of the service provided within the proposed systems.

\section{TABLE (1): COMPARING VIDEO STREAMING}

\begin{tabular}{|c|c|c|c|c|c|c|}
\hline Scheduling & $>$ & $z$ & $z$ & z & $z$ & $>$ \\
\hline $\begin{array}{l}\text { Computation } \\
\text { Element }\end{array}$ & 咅 & 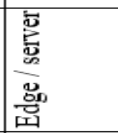 & 蓠 & 悹 & 离 & 悹 \\
\hline Type of user & 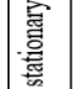 & 을 & 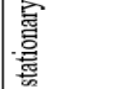 & 总 & 을 & 을 \\
\hline $\begin{array}{c}\text { QoS } \\
\text { Management }\end{array}$ & $z$ & $z$ & $>$ & $>$ & $>$ & $z$ \\
\hline Mobility & $>$ & $\Rightarrow$ & $>$ & $>$ & $\Rightarrow$ & $>$ \\
\hline Scalability & $>$ & $>$ & $>$ & $>$ & $>$ & $>$ \\
\hline Heterogeneit & $>$ & $>$ & $>$ & $>$ & $>$ & 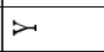 \\
\hline Media & 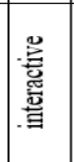 & 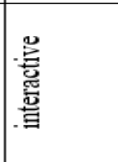 & 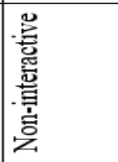 & 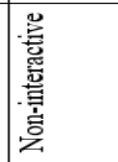 & 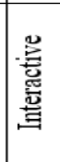 & 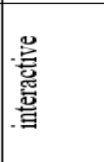 \\
\hline $\begin{array}{l}\text { Technology } \\
\text { Used }\end{array}$ & 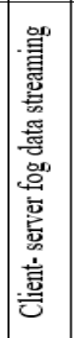 & 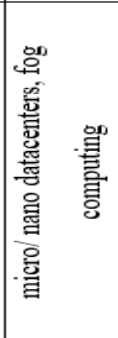 & 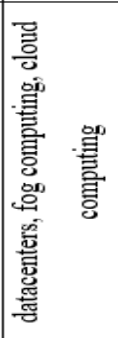 & 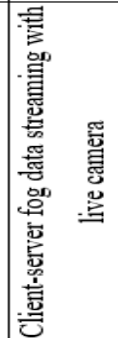 & 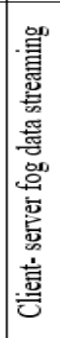 & 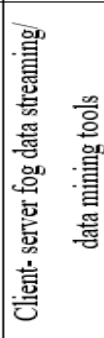 \\
\hline Reference & $\bar{m}$ & $\Phi$ & 共 & 槿 & 寻 & 急 \\
\hline
\end{tabular}




\section{REFERENCES}

[1] Hussain, Md, and M. M. Beg. "Fog Computing for Internet of Things (IoT)-Aided Smart Grid Architectures." Big Data and Cognitive Computing 3.1 (2019): 8.

[2] Luan, Tom H., et al. "Fog computing: Focusing on mobile users at the edge." arXiv preprint arXiv:1502.01815 (2015).

[3] Mahmud, Redowan, Ramamohanarao Kotagiri, and Rajkumar Buyya "Fog computing: A taxonomy, survey and future directions." Internet of everything. Springer, Singapore, 2018. 103-130.

[4] Peng, Mugen, et al. "Fog computing based radio access networks: Issues and challenges." arXiv preprint arXiv:1506.04233 (2015).

[5] Dastjerdi, Amir Vahid, and Rajkumar Buyya. "Fog computing: Helping the Internet of Things realize its potential." Computer 49.8 (2016): 112-116.

[6] Pecori, Riccardo. "A virtual learning architecture enhanced by fog computing and big data streams." Future Internet 10.1 (2018): 4.

[7] Concolato, Claude E., and Li M. Chen. "Data science: A new paradigm in the age of big-data science and analytics." New Mathematics and Natural Computation 13.02 (2017): 119-143.

[8] Pecori, Riccardo. "A virtual learning architecture enhanced by fog computing and big data streams." Future Internet 10.1 (2018): 4.

[9] Hashem, Ibrahim Abaker Targio, et al. "The rise of "big data" on cloud computing: Review and open research issues." Information systems 47 (2015): 98-115.

[10] Chen, CL Philip, and Chun-Yang Zhang. "Data-intensive applications, challenges, techniques and technologies: A survey on Big Data." Information sciences 275 (2014): 314-347.

[11] Wu, Xindong, et al. "Data mining with big data." IEEE transactions on knowledge and data engineering 26.1 (2014): 97-107.

[12] Hanson, Jeffery A., and Haw Yang. "Quantitative evaluation of cross correlation between two finite-length time series with applications to single-molecule FRET." The Journal of Physical Chemistry B 112.44 (2008): 13962-13970.

[13] Khan, Nawsher, et al. "Big data: survey, technologies, opportunities, and challenges." The Scientific World Journal 2014 (2014)

[14] Costan, Alexandru. From Big Data to Fast Data: Efficient Stream Data Management. Diss. ENS Rennes, 2019.

[15] Kaisler, Stephen, et al. "Big data: Issues and challenges moving forward." 2013 46th Hawaii International Conference on System Sciences. IEEE, 2013.

[16] Grolinger, Katarina, et al. "Challenges for mapreduce in big data." 2014 IEEE world congress on services. IEEE, 2014.

[17] Jaseena, K. U., and Julie M. David. "Issues, challenges, and solutions: big data mining." CS \& IT-CSCP 4.13 (2014): 131-140.

[18] Nasser, T., and R. S. Tariq. "Big data challenges." J Comput Eng Inf Technol 4: 3. doi: http://dx. doi. org/10.4172/2324 9307.2 (2015).

[19] Bonomi, Flavio, et al. "Fog computing and its role in the internet of things." Proceedings of the first edition of the MCC workshop on Mobile cloud computing. ACM, 2012

[20] Stojmenovic, Ivan, and Sheng Wen. "The fog computing paradigm: Scenarios and security issues." 2014 Federated Conference on Computer Science and Information Systems. IEEE, 2014

[21] Yi, Shanhe, Cheng Li, and Qun Li. "A survey of fog computing: concepts, applications and issues." Proceedings of the 2015 workshop on mobile big data. ACM, 2015

[22] Tang, Bo, et al. "A hierarchical distributed fog computing architecture for big data analysis in smart cities." Proceedings of the ASE BigData \& SocialInformatics 2015. ACM, 2015.

[23] Dubey, Harishchandra, et al. "Fog data: Enhancing telehealth big data through fog computing." Proceedings of the ASE bigdata \& socialinformatics 2015. ACM, 2015.

[24] Bonomi, Flavio, et al. "Fog computing: A platform for internet of things and analytics." Big data and internet of things: A roadmap for smart environments. Springer, Cham, 2014. 169-186.

[25] Stojmenovic, Ivan, et al. "An overview of fog computing and its security issues." Concurrency and Computation: Practice and Experience 28.10 (2016): 2991-3005.

[26] Bellavista, Paolo, and Alessandro Zanni. "Feasibility of fog computing deployment based on docker containerization over raspberrypi." Proceedings of the 18th international conference on distributed computing and networking. ACM, 2017.

[27] Aazam, Mohammad, and Eui-Nam Huh. "Fog computing and smart gateway based communication for cloud of things." 2014 International Conference on Future Internet of Things and Cloud. IEEE, 2014.

[28] Yannuzzi, Marcelo, et al. "Key ingredients in an IoT recipe: Fog Computing, Cloud computing, and more Fog Computing." 2014 IEEE 19th International Workshop on Computer Aided Modeling and Design of Communication Links and Networks (CAMAD). IEEE 2014.

[29] Yi, Shanhe, et al. "Fog computing: Platform and applications." 2015 Third IEEE Workshop on Hot Topics in Web Systems and Technologies (HotWeb). IEEE, 2015

[30] Stojmenovic, Ivan. "Fog computing: A cloud to the ground support for smart things and machine-to-machine networks." 2014 Australasian Telecommunication Networks and Applications Conference (ATNAC). IEEE, 2014.

[31] Luan, Tom H., et al. "Fog computing: Focusing on mobile users at the edge." arXiv preprint arXiv:1502.01815 (2015)

[32] Jalali, Fatemeh, et al. "Fog computing may help to save energy in cloud computing." IEEE Journal on Selected Areas in Communications 34.5 (2016): 1728-1739.

[33] Aazam, Mohammad, and Eui-Nam Huh. "Fog computing: The cloudiotVioe middleware paradigm." IEEE Potentials 35.3 (2016): 40-44.

[34] Masip-Bruin, Xavi, et al. "Foggy clouds and cloudy fogs: a real need for coordinated management of fog-to-cloud computing systems." IEEE Wireless Communications 23.5 (2016): 120-128.

[35] Dinh, Hoang T., et al. "A survey of mobile cloud computing: architecture, applications, and approaches." Wireless communications and mobile computing 13.18 (2013): 1587-1611.

[36] Yi, Shanhe, Zhengrui Qin, and Qun Li. "Security and privacy issues of fog computing: A survey." International conference on wireless algorithms, systems, and applications. Springer, Cham, 2015.

[37] Hassan, Saba Fouad, and Rana Fareed. "Video streaming processing using fog computing." 2018 International Conference on Advanced Science and Engineering (ICOASE). IEEE, 2018.

[38] Bilal, Kashif, and Aiman Erbad. "Edge computing for interactive media and video streaming." 2017 Second International Conference on Fog and Mobile Edge Computing (FMEC). IEEE, 2017.

[39] Lai, Chin-Feng, et al. "A QoS-aware streaming service over fog computing infrastructures." 2016 Digital Media Industry \& Academic Forum (DMIAF). IEEE, 2016

[40] Chen, Ning, et al. "Dynamic urban surveillance video stream processing using fog computing." 2016 IEEE second international conference on multimedia big data (BigMM). IEEE, 2016.

[41] Cao, Hung, Monica Wachowicz, and Sangwhan Cha. "Developing an edge computing platform for real-time descriptive analytics." 2017 IEEE International Conference on Big Data (Big Data). IEEE, 2017.

[42] Ma, Bin Bin, Simon Fong, and Richard Millham. "Data stream mining in fog computing environment with feature selection using ensemble of swarm search algorithms." 2018 Conference on Information Communications Technology and Society (ICTAS). IEEE, 2018.

[43] Reem Razzaq Abdul Hussein, "ROLE OF DATA MINING IN EGOVERNMENT FRAMEWORK", Iraqi Journal for Computers and Informatics, Vol. 44, 2018, pp. 1-4. 